\section{Nonperiodic Lamellar Phase in Ternary Diblock Copolymer/Homopolymer Blends}

\section{J . R. Naughton and M. W. Matsen*}

Polymer Science Centre, University of Reading, Whiteknights, Reading RG6 6AF, United Kingdom

Received J une 17, 2002

Revised Manuscript Received September 27, 2002

One of the more important applications of $A B$ diblock copolymer molecules is the compatiblization of immiscible A + B homopolymer blends. The importance has become even more significant since experiments by Bates et al. ${ }^{1}$ demonstrated that diblock copolymers can actually stabilize immiscible homopolymers through the formation of bicontinuous microemulsion. Despite this, relatively little effort has been devoted toward mapping out the bulk phase behavior of ternary diblock copolymer/ homopolymer blends. The most notable efforts are the mean-field calculations by J anert and Schick ${ }^{2,3}$ and the fluctuation corrections by Kielhorn and Muthukumar. ${ }^{4}$ There are al so Monte Carlo simulations by Müller and Schick 5 for low molecular weights where fluctuation effects are particularly large.

Here, we revisit the mean-field ternary phase diagram for symmetric $A B$ diblock copolymer with equal amounts of equally sized A- and B-type homopolymers. Results will only be presented for the special case where the copolymer and homopolymers have the same number of segments, $\mathrm{N}$, although we do have analogous results for a range of other molecular weight ratios. (Note that we define the $A$ and $B$ segments to have equal volumes, $\rho_{0}^{-1}$, and we assume that they al so have equal statistical segment lengths, a.) J anert and Schick ${ }^{2}$ have previously calculated the phase diagram for this particular case using self-consistent-field theory (SCFT), and interestingly they predicted two asymmetric lamellar phases $\left(L_{A}\right.$ and $\left.L_{B}\right)$ in addition to the usual symmetric one $(L)$. However, their predictions turn out to be incomplete.

The corrected SCFT phase diagram is shown in Figure 1 plotted in terms of segregation, $\chi \mathrm{N}$, and copolymer vol ume fraction, $\phi$. The order-disorder transition (ODT) remains as predicted previously by Broseta and $\mathrm{F}$ redrickson 6 and includes a Lifshitz tricritical point at $\chi \mathrm{N}=6$ and $\phi=2 / 3$ investigated previously by Hølyst and Schick. ${ }^{7}$ Furthermore, the $L+A+B$ coexistence region at high segregation remains the same except that it terminates at a slightly different value of $\chi \mathrm{N}=11.171$. The significant revisions are that the $L_{A}+L_{B}$ coexistence, predicted by J anert and Schick, ${ }^{2}$ is now replaced by an $L+A+B$ coexistence region, and their $L+L_{A}+$ $L_{B}$ coexistence is replaced by a new nonperiodic lamellar phase $\left(L_{n p}\right)$ consisting of a random sequence of thin and thick domains. The $L_{n p}$ region terminates at $\chi \mathrm{N}=$ 10.675 due to the fact that its two domain sizes gradually merge with decreasing segregation. We attribute our corrections, in part, to a more thorough investigation of the $L_{n p}$ region and improved numerical accuracy.

Although Figure 1 is calculated with the full SCFT, the phase behavior is most easily understood by taking advantage of an approximation where the free energy of an arbitrary lamellar structure is described by a tension, $\sigma$, for each diblock monolayer and a pairwise

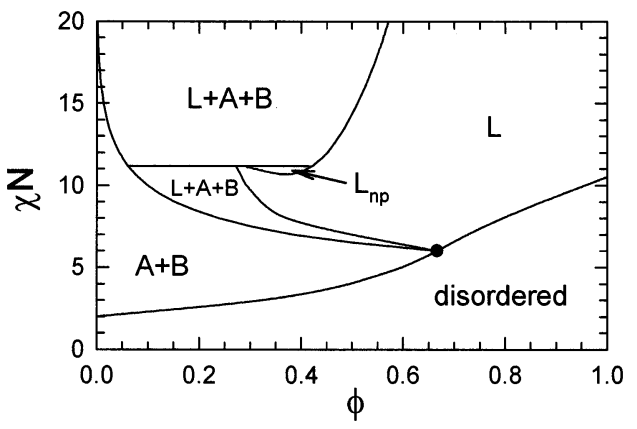

Figure 1. Mean-field phase diagram for symmetric ternary blends of $A B$ diblock copolymer, A homopolymer, and B homopolymer plotted as a function of segregation, $\chi \mathrm{N}$, and copolymer volume fraction, $\phi$. The diagram includes A-homopolymer-rich (A), B-homopolymer-rich (B), periodic lamellar $(L)$, and nonperiodic lamellar $\left(L_{n p}\right)$ ordered phases and one homogeneous disordered phase. The solid dot al ong the ODT denotes a Lifshitz tricritical point. ${ }^{7}$

interaction, $\mathrm{U}(\mathrm{d})$, between neighboring monolayers. More specifically, we approximate the free energy of a lamellar phase as

$$
\mathrm{F}_{\mathrm{L}}=\mathrm{F}_{\mathrm{A}+\mathrm{B}}+\mathrm{mA} \sigma+\mathrm{A} \sum_{\mathrm{i}=1}^{\mathrm{m}} \mathrm{U}\left(\left|\mathrm{z}_{\mathrm{i}+1}-\mathrm{z}_{\mathrm{i}}\right|\right)
$$

where $F_{A+B}$ is the free energy of a uniform homopolymer-rich phase, $\mathrm{m}$ is the total number of diblock monolayers, $\mathrm{A}$ is the area of each monolayer, and $\mathrm{z}_{\mathrm{i}}$ is the position of the ith monolayer (note that we assume periodic boundary conditions with $\mathrm{z}_{\mathrm{m}+1}=\mathrm{z}_{1}$ ). Equation 1 can be rewritten as

$$
\mathrm{F}_{\mathrm{L}}=\mathrm{F}_{\mathrm{A}+\mathrm{B}}+\sum_{\mathrm{i}=1}^{\mathrm{m}} \mathrm{F}_{0}\left(\mathrm{~d}_{\mathrm{i}}\right)
$$

where $d_{i}=\left|z_{i+1}-z_{i}\right|$ is the thickness of the ith domain and

$$
\frac{\mathrm{F}_{0}(\mathrm{~d})}{\mathrm{Ad}}=\frac{\sigma+\mathrm{U}(\mathrm{d})}{\mathrm{d}}
$$

is interpreted as the free energy density of a domain of thickness $d$. This decouples the free energy of the domains, allowing us to consider them independently.

Reference 8 describes the calculation of $\sigma$ and $\mathrm{U}(\mathrm{d})$ by examining the free energy of a pair of isolated diblock monolayers as a function of separation at fixed chemical potential and segregation. It is important to note that $\sigma$ is generally negative in the $L$ region and increases with the addition of homopolymer. As shown in Figure 2, U (d) generally has two minima: a well-pronounced one at $d=d_{\min , 1}$ and a very shallow one at $d=d_{\min , 2}$. The former minimum is primarily attributed to changes in the configurational entropy of the homopolymer. ${ }^{8} \mathrm{~F}$ or discussion purposes, we can ignore the weak dependence of $U$ (d) on chemical potential, but we must pay particular attention to the effect of segregation on the depth of the $d=d_{\min , 1}$ minimum

As demonstrated in Figure 2a, the equilibrium domain thickness minimizing $\mathrm{F}_{0}(\mathrm{~d}) / \mathrm{Ad}$ can be obtained graphically from the contact point where the lowest 

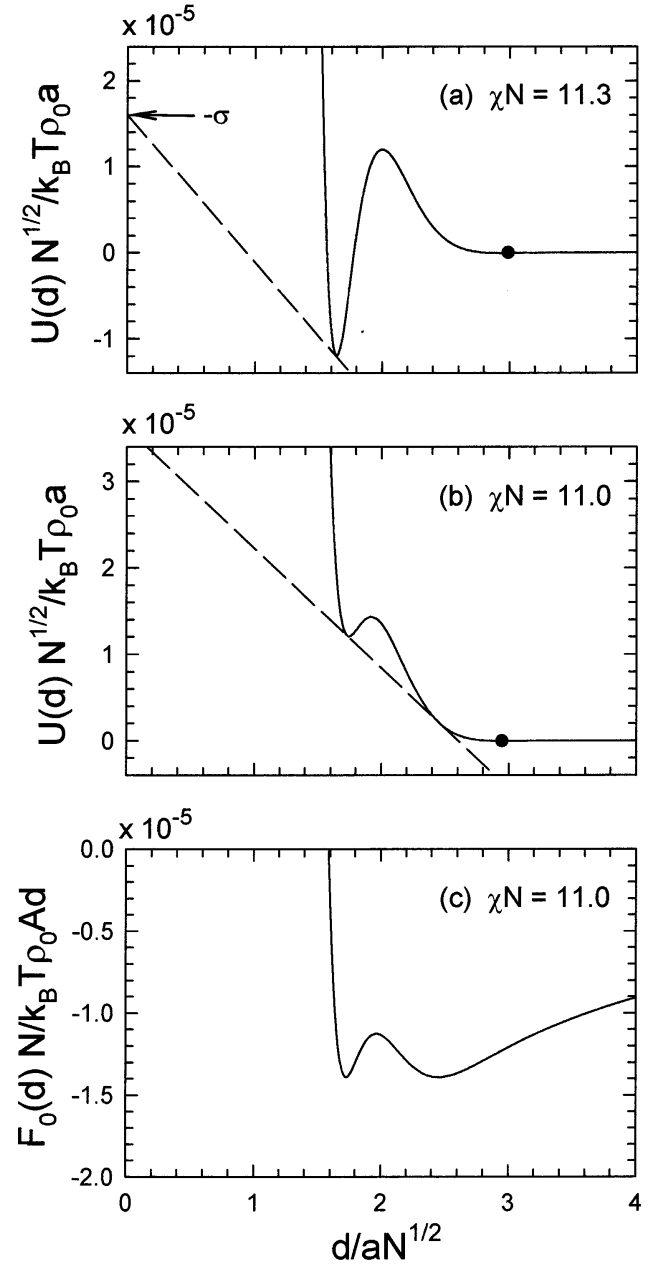

Figure 2. Pairwise interaction, $U(d)$, between two isolated monolayers as a function of their separation, $d$, calculated at (a) $\chi \mathrm{N}=11.3$ and (b) $\chi \mathrm{N}=11.0$ with chemical potentials corresponding to the $L+A+B$ and $L_{n p}$ regions, respectively. The solid dots denote the shallow minimum at $d=d_{\min , 2}$, and the dashed lines show tangent-line constructions for $\sigma=-1.6$ $\times 10^{-5} \mathrm{k}_{\mathrm{B}} \mathrm{T} \rho_{0} \mathrm{aN}-1 / 2$ and $\sigma=-3.62 \times 10^{-5} \mathrm{k}_{\mathrm{B}} \mathrm{T} \rho_{0} \mathrm{aN}-1 / 2$, respectively. (c) Free energy density, $\mathrm{F}_{0}(\mathrm{~d}) / \mathrm{Ad}$, of a domain as a function of thickness, $d$, calculated for the double-tangent line corresponding to the $L_{n p}$ region.

possible tangent line originating from the point $(-\sigma, 0)$ touches the $\mathrm{U}(\mathrm{d})$ curve. It follows that the main effect of adding homopolymer is to increase the equilibrium domain size by lowering the pivot point $(-\sigma, 0)$. When $\mathrm{U}\left(\mathrm{d}_{\min , 1}\right)<\mathrm{U}\left(\mathrm{d}_{\min , 2}\right)$, this occurs continuously until the tangent line becomes horizontal [i.e., $\sigma=-U\left(d_{\min , 1}\right)$ ], which corresponds to the onset of $\mathrm{L}+\mathrm{A}+\mathrm{B}$ coexistence according to eqs 2 and 3. Indeed, any higher value of $\sigma$ would cause the tangent line to make contact at $d=\infty$, which is equivalent to $A+B$ coexistence. When $U\left(d_{\min , 1}\right)$ $>U\left(d_{\min , 2}\right)$, the behavior is distinctly different in that the domain size makes a discontinuous jump from thin to thick. At the point of the transition illustrated by the double-tangent line in Figure $2 \mathrm{~b}$, there are two thicknesses of equal energy density resulting in the $L_{n p}$ morphology. As $\sigma$ increases further, a long-period $L$ phase occurs up to the point where the tangent line becomes horizontal [i.e., $\sigma=-\mathrm{U}\left(\mathrm{d}_{\mathrm{min}, 2}\right)$ ] as this again signifies $L+A+B$ coexistence.

The interpretation of the double-tangent line as a nonperiodic $L_{n p}$ phase requires the free energy of the individual domains to be uncoupled as predicted by the form of eq 1. To illustrate the accuracy of eq 1, we note
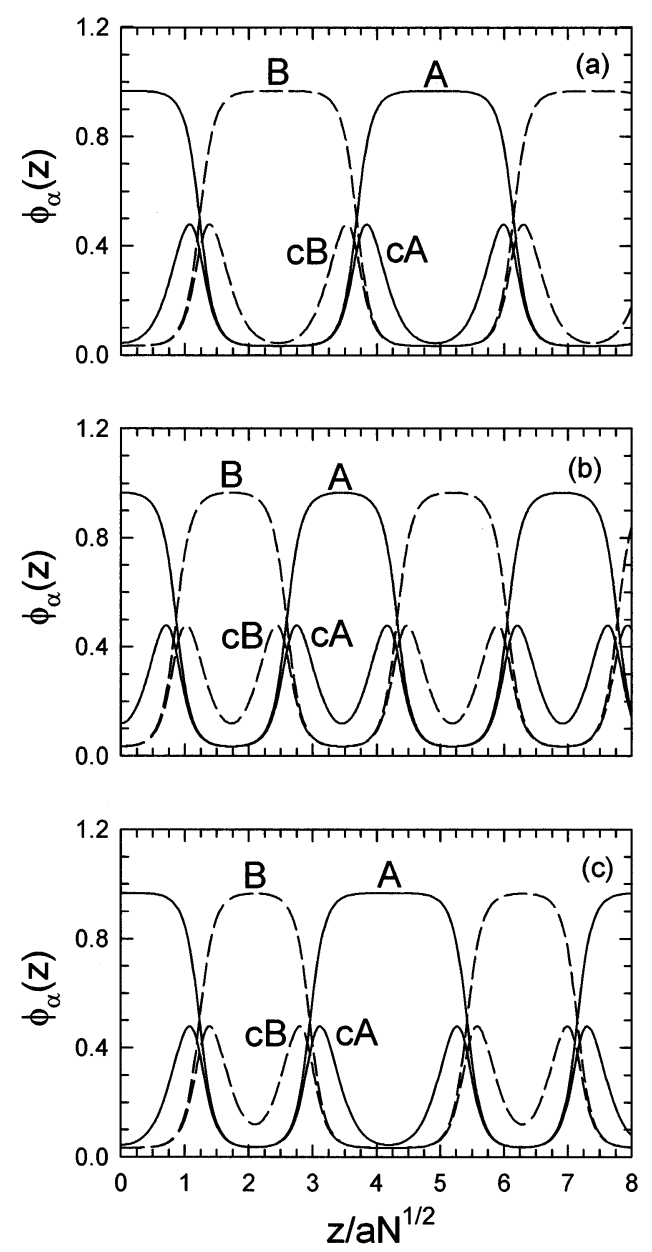

Figure 3. $A$ and $B$ segment profiles, $\phi_{A}(z)$ and $\phi_{B}(z)$, along with the copolymer contributions, $\phi_{\mathrm{CA}}(\mathrm{z})$ and $\phi_{\mathrm{CB}}(\mathrm{z})$, plotted for lamellar structures with (a) all-thick domains, (b) all-thin domains, and (c) alternating thin-thick domains, each calculated in the $L_{n p}$ region at $\chi N=11.0$. The solid and dashed curves denote the $A$ and $B$ components, respectively. N ote that the two domain sizes are given by the minima in Figure 2c.

that the free energy density, $\mathrm{F}_{0}(\mathrm{~d}) / \mathrm{Ad}$, of an isolated domain is completely indistinguishable, on the scale of Figure 2c, from the free energy per unit volume of a symmetric $L$ phase relative to the homopolymer-rich phases, $\left(F_{L}-F_{A+B}\right) N$, as calculated by the full SCFT. Furthermore, when we compare the long- and shortperiod symmetric $L$ phases with the asymmetric $L_{A}$ phase predicted by J anert and Schick ${ }^{2}$ at $\chi \mathrm{N}=11.0$ using the full SCFT, the difference in their free energies is less than $4 \times 10^{-9} \mathrm{k}_{\mathrm{B}} \mathrm{T}$ per molecule. Moreover, the $L_{A}$ phase has a period equal to the average of that of the two symmetric $L$ phases to within our numerical accuracy of 1 part in $10^{4}$. Careful examination of the segment profiles also reveals that the $A$ domains of the long-period $L$ phase (see Figure $3 a$ ) and the B domains of the short-period $L$ phase (see Figure $3 b$ ) are virtually indistinguishable from the $A$ and $B$ domains, respectively, of the $L_{A}$ phase (see Figure $3 c$ ).

Although the evidence above demonstrates that eq 1 is exceptionally accurate, there are nevertheless tiny corrections that will prefer certain lamellar sequences in the $L_{n p}$ region. For instance, we find that the symmetric (i.e., all-thin or all-thick) $L$ phases are slightly more stable than the alternating thin-thick $L_{A}$ phase. However, there is an infinite set of sequences to examine, and thus we could never identify the true equilibrium one. In any case, this becomes a purely 
academic issue given the tiny energy differences involved. When an actual system enters the $L_{n p}$ region, the interaction, $U(d)$, will dominate, causing the domains to first adopt one of the two preferred thicknesses with virtually no regard to the actual sequence, inevitably producing a nonperiodic $L_{n p}$ structure. Once this step is complete, the small energy corrections would, in principle, cause the system to slowly evolve toward the most preferred equilibrium sequence. However, considering the amount of homopolymer that has to be transported in order to alter the sequence and the incredibly small return in energy, the system is certain to be metastably trapped in the initial $\mathrm{L}_{n p}$ morphology.

Up to now we have ignored fluctuation effects, which become increasingly important for low molecular weights. On the basis of the weak variation in $\mathrm{U}(\mathrm{d})$ at large $\mathrm{d} \sim$ $d_{\min , 2}$ as well as experiment, ${ }^{1}$ we expect the lower $L+$ $A+B$ region in Figure 1 to be highly prone to fluctuations that would transform it into a bicontinuous microemulsion. On the other hand, the upper $L+A+$ $B$ region should be relatively stable against fluctuations given the deep minimum at $d=d_{m i n, 1}$. As evident from Figure 2, the $L_{n p}$ phase is well segregated and thus should also be reasonably stable with respect to fluctuations, although they may truncate the lower part of the $L_{n p}$ region where the two thicknesses become similar. Naturally, our findings extend beyond the particular case examined above. F or instance, all the ternary phase diagrams in ref 2 will have to be revised. Furthermore, the $L+L_{A}$ coexistence predicted for binary blends in refs 2 and 9 likely will be replaced by a $L_{n p}$ region. In other instances where lamellae were predicted to unbind, , 2,3,9-11 we instead expect macrophase separation. In fact, we have confirmed this expectation with calculations for a binary diagram in refs 2 and 9 and a ternary diagram in ref 10 . Our results for the later case were also confirmed by independent calculations while this Communication was in review. ${ }^{12}$
In conclusion, we have revised the phase diagram predicted by J anert and Schick ${ }^{2}$ for symmetric ternary blends of $A B$ diblock copolymer and A + B homopolymers. First, we predict a unique nonperiodic lamellar phase, $L_{n p}$, consisting of a random sequence of thin and thick domains. Second, we find that none of the lamellar phases unbind, but instead the homopolymer macrophase separates, forming a $L+A+B$ coexistence region. As a result of these findings, a number of previous predictions will have to be reconsidered.

Acknowledgment. This work was supported by the EPSRC (GR/M22130).

\section{References and Notes}

(1) Bates, F. S.; Maurer, W. W.; Lipic, P. M.; Hillmyer, M. A.; Almdal, K.; Mortensen, K.; Fredrickson, G. H.; Lodge, T. P. Phys. Rev. Lett. 1997, 79, 849.

(2) J anert, P. K.; Schick, M. Macromolecules 1997, 30, 3916.

(3) J anert, P. K.; Schick, M. Macromolecules 1997, 30, 137.

(4) Kielhorn, L.; Muthukumar, M. J . Chem. Phys. 1997, 107, 5588.

(5) Müller, M.; Schick, M. J . Chem. Phys. 1996, 105, 8885; the blends in this paper correspond to a small invariant polymerization index, $\mathrm{N} \equiv \mathrm{a}^{6} \rho_{0}{ }^{-2} \mathrm{~N}=3.05^{6} \times 16^{-2} \times 32=100$.

(6) Broseta, D.; Fredrickson, G. H. J . Chem. Phys. 1990, 93, 2927.

(7) Hølyst, R.; Schick, M. J . Chem. Phys. 1992, 96, 7728.

(8) Thompson, R. B.; Matsen, M. W. J . Chem. Phys. 2000, 112, 6863.

(9) J anert, P. K.; Schick, M. Phys. Rev. E 1996, 54, R33; Macromolecules 1998, 31, 1109.

(10) Bates, F. S.; Maurer, W.; Lodge, T. P.; Schulz, M. F.; Matsen, M. W.; Almdal, K.; Mortensen, K. Phys. Rev. Lett. 1995, 75, 4429.

(11) Matsen, M. W. Phys. Rev. Lett. 1995, 74, 4225; Macromolecules 1995, 28, 5765.

(12) Duechs, D., private communication.

MA025580U 